\title{
ARTÍCULOS
}

Sometido 02.08.2015. Aprobado 18.04.2016

Evaluado por el sistema double blind review. Editor Científico: Eric Cohen

DOI: http://dx.doi.org/10.1590/So034-759020160507

\section{EVALUACIÓN DE LA DISTRIBUCIÓN ESPACIAL DE PLANTAS INDUSTRIALES MEDIANTE UN ÍNDICE DE DESEMPEÑO}

\author{
Avaliação da distribuição espacial de plantas industriais segundo um índice \\ de desempenho
}

An approach to industrial facility layout evaluation using a performance index

\begin{abstract}
RESUMEN
Una amplia literatura científica ha abordado el problema de la distribución espacial de plantas industriales completamente nuevas. Sin embargo, no se ha prestado suficiente atención al problema de la redistribución de instalaciones ya existentes. Las escasas investigaciones que evalúan el layout, en su mayoría, aplican métodos que solo tienen sentido si se comparan varias alternativas. En consecuencia, no son de utilidad para evaluar el desempeño del layout actual de una planta industrial y no permiten diagnosticar la necesidad de una redistribución. Frente a esto, este trabajo presenta un indicador que permite evaluar el desempeño de una distribución espacial existente, identificando su posicionamiento entre el escenario ideal y el anti-ideal. De tal forma, permite sustentar la pertinencia o irrelevancia de una redistribución. El indicador fue aplicado en el caso práctico de una empresa del sector metalmecánico de la ciudad de Guayaquil.
\end{abstract}

PALABRAS CLAVE | Índice de desempeño del layout, distribución en planta, evaluación de la distribución en planta, redistribución, empresa metalmecánica.

\section{RESUMO}

Uma ampla literatura científica tem abordado o problema da distribuição espacial de plantas industriais totalmente novas. No entanto, não se tem prestado atenção suficiente ao problema da redistribuição de instalações já existentes. Em sua maioria, as poucas investigações que avaliam o layout aplicam métodos que só fazem sentido quando se comparam várias alternativas. Consequentemente, não são úteis para analisar o desempenho do layout atual de uma planta industrial e não permitem diagnosticar a necessidade de uma redistribuição. Levando isso em consideração, este trabalho apresenta um indicador que permite dimensionar o desempenho de uma distribuição espacial existente, identificando seu posicionamento entre o cenário ideal e o não ideal. Dessa maneira, permite sustentar a pertinência ou a irrelevância de uma redistribuição. $O$ indicador foi aplicado ao caso prático de uma empresa do setor metal-mecânico da cidade de Guayaquil.

PALAVRAS-CHAVE I Índice de desempenho do layout, distribuição em planta, avaliação da distribuição em planta, redistribuição, empresa metal-mecânica.

\begin{abstract}
A good deal of literature dealing with the facility layout problem has focused on greenfield layout design. However, little attention has been paid to the facility re-layout problem. Generally, methods applied to plant layout evaluation research, only make sense if several different layout alternatives are compared. Consequently, these methods tend to have limited value in evaluating existing plant layouts or in supporting the need for a re-layout. With this in mind, this work suggests an indicator that allows one to evaluate an existing plant's layout performance by identifying where it stands in relation to the ideal and the non-ideal scenarios. The application of this particular indicator can help justify the pertinence or irrelevance of facility re-layout decisions. The method is illustrated in a case study carried out on a company in the metal components processing industry, located in the city of Guayaquil.
\end{abstract}

KEYWORDS I Layout performance index, facility layout, facility layout evaluation, facility re-layout, metal components processing industry.

PABLO ALBERTO PÉREZ GOSENDE pperezg@ups.edu.ec

Profesor de la Universidad

Politécnica Salesiana del EcuadorGuayaquil, Ecuador 


\section{INTRODUCCIÓN}

La distribución en planta o layout, es el proceso de ordenamiento de los elementos que conforman el sistema productivo en el espacio físico, de manera que se alcancen los objetivos de producción de la forma más adecuada y eficiente posible. Es considerada una de las decisiones de diseño más importantes dentro de la estrategia de operaciones de una organización (Chase \& Jacobs, 2014; Krajewski, Malhotra, \& Ritzman, 2015).

La distribución en planta tiene un impacto importante y directo en la eficiencia de la producción y el nivel de productividad de los sistemas de manufactura (Ariafar, Ismail, Tang, Ariffin, \& Firoozi, 2011; Edis, Kahraman, Araz, \& Özfirat, 2011; GarciaHernandez, Arauzo-Azofra, Salas-Morera, Pierreval, \& Corchado, 2013; Kanaganayagam, Muthuswamy, \& Damodaran, 2015; Mallikarjuna, Veeranna, \& Reddy, 2011; Ripon \& Torresen, 2014; Sadeghpour \& Andayesh, 2015; Vasudevan \& Son, 2011). De ahí que la identificación de las oportunidades de mejora de la distribución espacial de los centros de actividad que conforman un sistema productivo sea vital para contribuir a la mejora de la productividad del trabajo de una organización.

De acuerdo con la Teoría de la Complejidad, el problema de la distribución en planta (Facility Layout Problem [FLP]) se clasifica como NP-completo (Garey \& Johnson, 1979). Los problemas clasificados bajo esta denominación, según Diego-Mas (2006), son considerados los más difíciles de resolver, pues no existe un algoritmo que proporcione una solución óptima en un tiempo razonable.

Este nivel de complejidad del FLP, dada su naturaleza multicriterio y multiobjetivo, no ha sido impedimento para que distintos autores lo hayan abordado aplicando desde técnicas exactas hasta las más avanzadas técnicas metaheurísticas, proporcionando soluciones aceptables en tiempos de cálculo realistas. Tales enfoques de resolución han sido aplicados, generalmente, al caso de plantas completamente nuevas. Sin embargo, a pesar de su poca trascendencia en la literatura, en la práctica el problema de la redistribución espacial de la planta (Facility Re-layout Problem [FRLP]) es más común (Kulturel-Konak, 2007).

Las empresas necesitan adaptarse constantemente a las necesidades cambiantes de los mercados. Para esto, aumentan o contraen su capacidad productiva, cambian parcial o totalmente de tecnología, crean nuevos productos y servicios y mejoran e implementan nuevos procesos. Esta dinámica requiere que las empresas dispongan de distribuciones espaciales suficientemente flexibles (Emami \& Nookabadi, 2013), pues de forma paulatina van perdiendo la bondad de la distribución en planta inicial y comienzan a suscitarse una serie de situaciones que pueden traer consigo la necesidad de una redistribución. De acuerdo con Chase y Jacobs (2014) y Krajewski et al. (2015), entre estos síntomas es probable encontrar simultaneidad de cuellos de botella, congestión y deficiente utilización del espacio, acumulación excesiva de materiales en proceso, puestos de trabajo ociosos o sobrecargados, ansiedad y malestar de la mano de obra, trabajadores calificados realizando demasiadas operaciones poco complejas, accidentes laborales y dificultad en el control de las operaciones y del personal.

A los síntomas anteriormente expuestos, habría que adicionar el exceso de manutención de materiales. Cuando no existe un adecuado nivel de adyacencia entre los centros de actividad de la organización, se genera un desaprovechamiento de la jornada laboral en actividades de transporte que no aportan valor. Esta es una de las razones principales por las que aumentan los tiempos de fabricación unitarios (throughput time) y disminuyen los niveles de productividad del trabajo.

La necesidad de una redistribución se presenta, entonces, cuando se detectan reservas de mejora de la productividad como resultado de un proceso de evaluación del layout. A pesar de la amplia literatura científica que aborda el FLP, son escasas las investigaciones que abordan esta etapa de evaluación, y no existen referencias de algún procedimiento que permita diagnosticar objetivamente el problema de la redistribución espacial.

La solución del FRLP es tanto o más compleja que la distribución espacial para una planta completamente nueva, dado que existen restricciones y objetivos adicionales. Implementar cambios en un layout existente requiere de inversión adicional y retrasos o interrupción total de los planes de producción durante el período que dure la redistribución. Sin embargo, dado que los cambios en el layout tienen un efecto significativo en la productividad, las decisiones de mejora de la distribución espacial son vitales para mantener un adecuado nivel de competitividad de la organización (Han, Bae, \& Jeong, 2012). De ahí la importancia de que las empresas cuenten con una herramienta que les permita evaluar el desempeño de su distribución actual. De esta manera podría diagnosticarse la necesidad de una redistribución de la planta, al identificarse oportunidades de mejora que podrían redundar en reservas de productividad.

Con este antecedente, en esta investigación se presenta un Índice de Desempeño del Layout (IDL) mediante el cual los gerentes de operaciones pueden evaluar el desempeño actual de la distribución espacial de la organización y sustentar la pertinencia o irrelevancia de una redistribución. El IDL permite identificar las reservas de mejora del layout determinando su posicionamiento entre el escenario ideal (que supone adyacencia entre los centros de actividad que tienen flujo de trabajo y/o relaciones cualitativas de cercanía entre sî), y el anti-ideal (aquel donde los centros de actividad están dispuestos de forma caótica en el espacio fabril, sin ningún tipo de relación cualitativa y/o cuantitativa que justifique su adyacencia). 


\section{MARCO TEÓRICO}

La generación y selección de la distribución espacial óptima para una organización es un proceso complejo e iterativo que depende de las relaciones que existen entre los elementos que conforman su sistema de producción de bienes o servicios. En el campo de la ingeniería, varias han sido las estrategias formuladas para resolver el problema de la distribución en planta. Entre ellas, de acuerdo con Diego-Mas (2006), las más importantes son las propuestas por Immer (1953), Buffa (1955), Muther (1961), Reed (1961), Nadler (1967) y Apple (1977) (referidas en DiegoMas, 2006). Sin embargo, el SLP (Systematic Layout Planning) creado por Muther (1961), ha sido la más aceptada y utilizada (Río-Cidoncha, Martínez-Palacios, \& Iglesias, 2007).

La mayoría de los estudios posteriores se han centrado en la fase de distribución de conjunto del SLP, que busca determinar el ordenamiento óptimo de $\mathrm{n}$ centros de actividad indivisibles en $\mathrm{n}$ posiciones dentro del espacio físico disponible, de forma tal que se satisfagan ciertos objetivos relevantes. Entre estos objetivos el más comúnmente utilizado, de acuerdo con Moslemipour, Lee, y Rilling (2012), es minimizar el costo de manutención de materiales. Sin embargo, otros objetivos tales como reducir la distancia recorrida por los materiales, disminuir los niveles de trabajo en proceso (work in progress) y los tiempos de fabricación (throughput times), y evitar la congestión general, también han sido considerados (Askin \& Standridge, 1993; Francis, McGinnis, \& White, 1992; Fu \& Kaku, 1997; Mecklenburgh, 1985).

El FLP ha sido modelado matemáticamente como un problema de asignación cuadrática (QAP) (Koopmans \& Beckman, 1957), de cobertura cuadrática de conjuntos (QSP) (Bazaraa, 1975), mediante programación entera lineal (LIP) (Lawler, 1963) y a través de programación entera mixta (MIP) (Kaufman \& Broeckx, 1978). También ha sido formulado mediante teoría de grafos (Foulds \& Robinson, 1978).

De manera general, los métodos usados en la búsqueda de soluciones óptimas o cercanas al óptimo, pueden categorizarse en métodos exactos, heurísticos y metaheurísticos (Matai, Singh, \& Mittal, 2010). Los métodos exactos como el Branch-and-bound y los algoritmos de corte han permitido obtener soluciones óptimas al FLP formulado como QAP, cuando el número de instalaciones es menor de 16 (Singh \& Sharma, 2006). Para un número mayor, el FLP no puede ser resuelto de forma óptima en un tiempo computacional razonable (Suo, 2012).

Para obtener soluciones cercanas al óptimo en un tiempo computacional razonable fueron desarrollados los métodos heurísticos. Básicamente, un algoritmo heurístico es un conjunto establecido de pasos que permite encontrar soluciones de buena calidad (Matai et al., 2010). De acuerdo con Moslemipour et al.
(2012), estos pueden ser clasificados en dos clases: algoritmos de construcción y algoritmos de mejora.

Los algoritmos de construcción son considerados los enfoques heurísticos más simples y antiguos para resolver el modelo QAP (Singh \& Sharma, 2006). Estos consisten en la sucesiva selección y colocación de centros de actividad en el plano de distribución, buscando minimizar el costo total de manutención de materiales. Según Matai et al. (2010), algunos de los algoritmos de construcción más conocidos en la literatura son: HC66 propuesto por Hillier y Connors (1966), ALDEP desarrollado por Seehof y Evans (1967) y CORELAP presentado por Lee y Moore (1967) (todos referidos en Matai et al., 2010). Lamentablemente, la simplicidad de estos algoritmos es frecuentemente asociada a soluciones de baja calidad (Singh \& Sharma, 2006).

Los algoritmos de mejora, por su parte, parten de una solución inicial que es mejorada mediante alguno de los siguientes enfoques: intercambiando la ubicación de un par de centros de actividad a la vez (pair-wise exchange) o intercambiando múltiples pares (multi-pair-wise exchange) (Moslemipour et al., 2012). En este caso, la calidad de la solución obtenida depende del layout inicial y el enfoque de intercambio elegido. Entre los algoritmos de mejora referenciados en la literatura se encuentran: CRAFT, creado por Armoury Buffa (1963); MULTIPLE, desarrollado por Bozer, Meller, y Erlebacher (1994); SABLE, creado por Meller y Bozer (1996); y MSA, desarrollado por Singh y Sharma (2006) (todos referidos en Matai et al., 2010).

En los últimos años, varios enfoques metaheurísticos como el Recocido Simulado (SA), Algoritmos Genéticos (GA), Enjambre de partículas (PSO), Búsqueda Tabú (TS) y Colonias de Hormigas (ACO), han sido aplicados exitosamente en la resolución de problemas de distribución de plantas industriales. Una revisión bastante exhaustiva de estos enfoques puede consultarse en Sharma, Singh y Singhal (2013).

Han et al. (2012), clasificaron el FLP en dos áreas fundamentales: la distribución de plantas completamente nuevas (Green field layout design) y la redistribución de plantas ya existentes (Facility re-layout problem, FRLP).

Un análisis de las investigaciones que han abordado el estado del arte del FLP (Drira, Pierreval, \& Hajri-Gabouj, 2007; Kulturel-Konak, 2007; Kusiak \& Heragu, 1987; Matai et al., 2010; Meller \& Gau, 1996; Moslemipour et al., 2012; Singh \& Sharma, 2006), permite concluir que la mayor parte de la literatura se enfoca en la distribución de plantas completamente nuevas. En tales casos, se busca diseñar la distribución espacial de una planta sin la influencia de las restricciones que normalmente se presentan al hacerlo en una instalación ya existente. Solo KulturelKonak (2007) señaló al problema de la redistribución espacial (FRLP) como un área por abordar en futuras investigaciones. 
Una redistribución de la planta, es la decisión que se toma como resultado de un proceso de evaluación, en el que se detecta que el layout no permite cumplir los objetivos trazados por la organización. En la literatura son escasas las investigaciones que evalúan la distribución espacial de instalaciones de producción o servicios. De hecho, en las últimas dos décadas, los enfoques del FLP se han centrado en la generación de alternativas de layout y muy pocos logros se han obtenido en la etapa de evaluación (McKendall, Shang, \& Kuppussamy, 2006; Shahin \& Poormostafa, 2011).

En este sentido, Neumann y Fogliatto (2013) propusieron un índice general para medir la flexibilidad del layout (IDFL) en entornos dinámicos, a partir de la medición de tres factores primarios, 12 secundarios, 33 terciarios y 50 indicadores de tipo cualitativo. Tortorella y Fogliatto (2008) combinaron herramientas de decisión multicriterio con SLP en la búsqueda de la mejor alternativa de layout para una empresa del sector automovilístico. La evaluación de alternativas en este caso se realizó mediante la técnica AHP (Analytic Hierarchy Process) basado en siete criterios: maximizar la flexibilidad para futuras expansiones, los factores humanos, el aprovechamiento del área, la linealidad del flujo, las relaciones entre centros de actividad y minimizar los reajustes y sus costos.

Shahin y Poormostafa (2011), utilizaron SLP para generar un número considerable de alternativas de layout, que luego fueron evaluadas mediante criterios cuantitativos y cualitativos a través de simulación y AHP difuso, respectivamente. El escenario más eficiente fue identificado mediante el método TOPSIS (Technique for Order of Preference by Similarity to Ideal Solution).

Yang, Su, y Hsu (2000), aplicaron SLP para generar alternativas de layout de una fábrica de obleas semiconductoras, que luego fueron evaluadas mediante AHP. Distribuciones celulares basadas en SLP fueron evaluadas mediante AHP por Nagapak y Phruksaphanrat (2011), en el caso de estudio de una planta de servicios de fabricación de componentes electrónicos. Combinaciones de AHP y DEA (Data Envelop Analysis) también han sido empleadas en la selección de la mejor distribución espacial (Gao, Yoshimoto, \& Ohmori, 2010; Yang \& Kuo, 2003).

Yang y Deuse (2012) utilizaron AHP para obtener las ponderaciones de los criterios de decisión y PROMETHEE (Preference Ranking Organization METHod for Enrichment of Evaluations) para seleccionar el mejor layout entre un grupo de alternativas. Önüt, Kara, y Efendigil (2008) desarrollaron un enfoque multicriterio híbrido basado en AHP difuso y TOPSIS difuso, para evaluar y seleccionar el layout para un centro de mecanizado CNC.

Como puede observarse, en la literatura de forma frecuente se han utilizado métodos de decisión multicriterio
(Multiple Criteria Decision Making, MCDM) para la evaluación de alternativas de distribución en planta. Sin embargo, a pesar de su extendido uso, las técnicas de MCDM ofrecen únicamente medidas relativas que solo tienen sentido si se comparan varias alternativas de layout. Por tal razón, no son de utilidad para evaluar el desempeño del layout actual de una planta industrial, o lo que es lo mismo, no permiten diagnosticar la necesidad de una redistribución.

De tal manera, el IDL se presenta en esta investigación como una herramienta que permite diagnosticar la necesidad de una redistribución espacial, a la vez que constituye un método de evaluación de layouts alternativo a los complejos métodos de decisión que involucran interacción humana como los métodos de decisión multicriterio.

\section{PRESENTACIÓN DEL ÍNDICE DE DESEMPEÑO DEL LAYOUT}

Se estableció un procedimiento de cinco pasos para la determinación del Índice de desempeño del Layout (IDL), mediante el cual se evaluará el desempeño de la distribución espacial actual de una organización. El esquema del procedimiento propuesto se presenta en la Figura 1.

\section{Figura 1. Procedimiento para el cálculo del Î́ndice de Desempeño del Layout (IDL)}

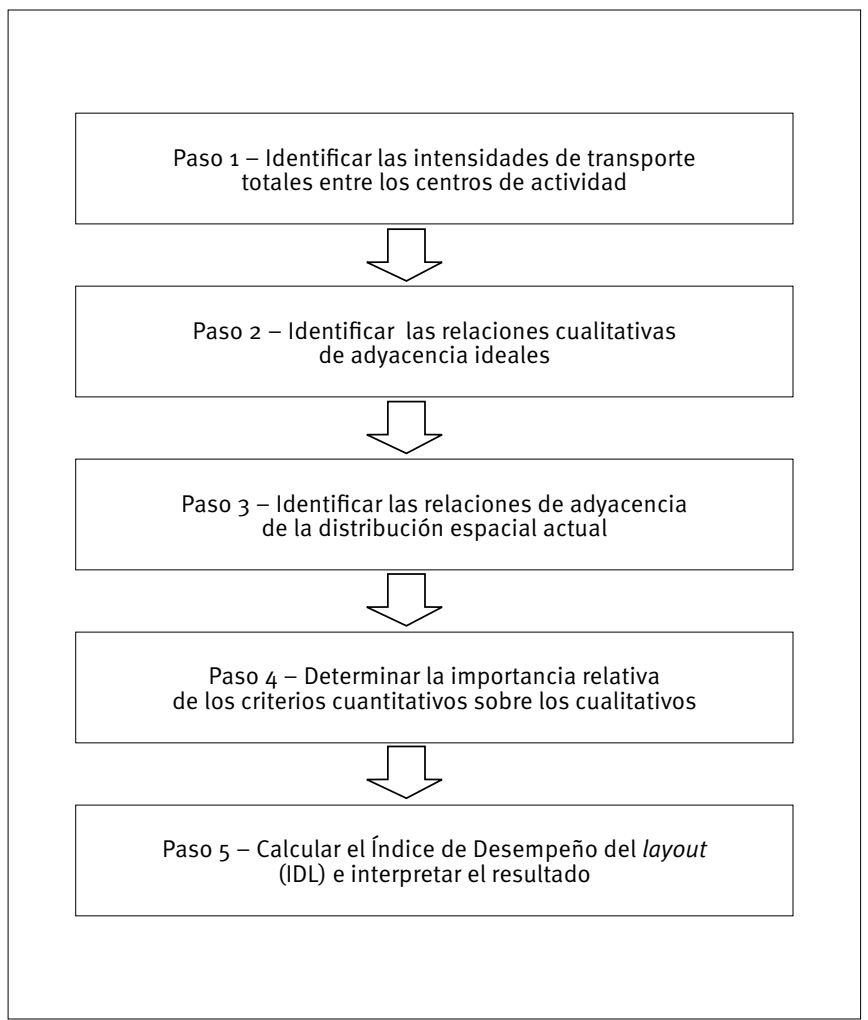


A continuación se describe el procedimiento con mayor detalle.

\section{Paso 1: Identificar las intensidades de transporte totales entre los centros de actividad}

La intensidad de transporte entre dos centros de actividad ( $\left.\mathrm{t}_{\mathrm{ij}}\right)$ corresponde a la cantidad de materiales (objeto de trabajo) que se traslada entre ellos en una unidad de tiempo. La unidad de medida de esta variable podría expresarse en l/día, $\mathrm{m}^{3} / \mathrm{mes}$, $\mathrm{t} / \mathrm{a} \tilde{n}$, entre otras, de acuerdo con las unidades de medidas apropiadas según el sistema productivo en estudio.

Evidentemente, no se espera obtener un valor numérico que exprese la intensidad de transporte para cada par posible de centros de actividad, pues estos no necesariamente mantienen un flujo de materiales entre sí. Lo anterior puede demostrarse en el caso de empresas que brindan servicios a clientes, sean estos servicios puros, un servicio principal con bienes y servicios secundarios, productos híbridos o bienes tangibles que incluyen algunos servicios (Kotler \& Keller, 2012). Aquí el flujo de trabajo no se referiría al material exclusivamente, como ocurre en sistemas de manufactura, sino a los clientes e incluso a la información.

Cuando el flujo de los clientes que se atienden es importante, la media de clientes que se desplazan entre los centros de actividad en una unidad de tiempo relevante, tal como una semana o un mes de operaciones, podría ser la intensidad de transporte adecuada a considerar. Sin embargo, cuando únicamente existe un flujo de información entre los centros de actividad, si este se realiza con soporte en las Tecnologías de la Información y las Comunicaciones (TIC), entonces no sería relevante considerar las intensidades de transporte como condicionante de la cercanía entre departamentos o áreas de actividad, sino el comportamiento de otros criterios de naturaleza cualitativa.

El primer paso del procedimiento involucra tres etapas que se describen a continuación.

\section{Etapa 1.1: Identificar los centros de actividad de la organización}

Un centro de actividad es un espacio físico en el que se realizan actividades involucradas en los procesos operativos, estratégicos y de apoyo de la organización, incluyendo los espacios necesarios para los servicios auxiliares. Pueden tener una frontera física o imaginaria, y su cantidad está en dependencia de la distribución básica escogida por la gerencia de la organización: por posición fija, por producto, por procesos o celular (Chase \& Jacobs, 2014; Krajewski et al., 2015). Los centros de actividad pueden identificarse mediante revisiones de los planos de planta, entrevistas a expertos u observación directa.

\section{Etapa 1.2: Desarrollar la matriz cuadrada orientada de las intensidades de transporte parciales (matriz T)}

La matriz cuadrada orientada de las intensidades de transporte parciales, denominada alternativamente matriz $\mathrm{T}$, tendrá un orden que corresponde al número de centros de actividad (n) que esté bajo consideración. Para su obtención, es necesario determinar la cantidad de materiales que se transporta entre cada par de centros de actividad en una unidad de tiempo apropiada de acuerdo a la estacionalidad de la demanda del producto, aunque utilizar un promedio mensual o anual, en cualquier caso, es siempre una buena estrategia. Esta información podría recopilarse de los registros de actividad que se tengan en la organización, o en última instancia, mediante observación directa.

$$
t=\left[\begin{array}{cccccc}
0 & t_{12} & t_{13} & t_{14} & \cdots & t_{1 n} \\
t_{21} & 0 & t_{23} & t_{24} & \cdots & t_{2 n} \\
t_{31} & 0 & 0 & t_{34} & \cdots & t_{3 n} \\
t_{41} & 0 & 0 & 0 & \cdots & t_{4 n} \\
\vdots & \vdots & \vdots & \vdots & \ddots & \vdots \\
t_{n 1} & t_{n 2} & t_{n 3} & t_{n 4} & \cdots & 0
\end{array}\right]
$$

\section{Etapa 1.3: Desarrollar la matriz triangular no orientada de las intensidades de transporte totales (matriz T)}

Aquí se busca obtener el flujo total de transporte entre cada par de centros de actividad en ambas direcciones. Esto se logra considerando que $\mathrm{T}_{\mathrm{ji}}=\mathrm{t}_{\mathrm{ij}}+\mathrm{t}_{\mathrm{ji}}$. El resultado es una matriz triangular no orientada de las intensidades de transporte totales a la que alternativamente se denominará matriz T.

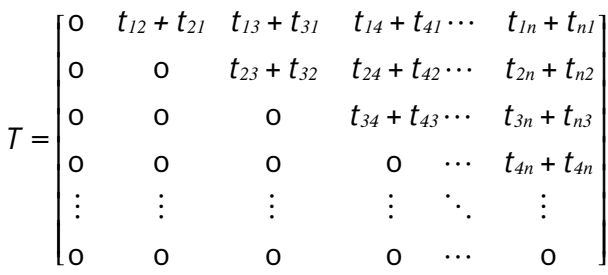




\section{Paso 2: Identificar las relaciones cualitativas de adyacencia ideales entre los centros de actividad}

Las relaciones existentes entre cada par de centros de actividad no deben limitarse a la intensidad del transporte de flujo de materiales o personas entre ellos, pues en ocasiones estas relaciones son irrelevantes o inexistentes. Es por esto que deben cuantificarse también las relaciones cualitativas que pudieran existir entre los centros de actividad que justifican las necesidades de adyacencia o su indeseabilidad. Entre estos, las exigencias constructivas, ambientales, de seguridad e higiene, los sistemas de manipulación necesarios, el abastecimiento de energía y la evacuación de residuos, la organización de la mano de obra, los sistemas de control del proceso, los sistemas de información, entre otros, podrían considerarse relevantes.

La valoración de las relaciones de adyacencia ideales de acuerdo con los criterios de naturaleza cualitativa entre los centros de actividad i y j se obtendrá mediante el juicio de expertos. Algunos autores defienden la utilidad del uso de expertos en la toma de decisiones cuando, como en este caso, se necesita obtener información precisa que no está disponible o es costosa de obtener; cuando se requiere estudiar o definir áreas donde hay una considerable incertidumbre y/o falta de consenso, y también para modelar un fenómeno del mundo real que implica una serie de puntos de vista y para el cual existe poca evidencia cuantitativa (Bendaña, Caño, \& Cruz, 2008; Bradley \& Stewart, 2002; Lucko \& Rojas, 2010; Orndoff, 2005; Padel \& Midmore, 2005; Sourani \& Sohail, 2014; Yeung, Chan, \& Chan, 2009). Otra de las bondades que saltan a la vista de forma evidente, es que el número de factores que es considerado por un grupo es mayor que el que podría ser tenido en cuenta por una sola persona. Cada experto podrá aportar a la discusión general la idea que tiene sobre el tema debatido desde su área de conocimiento. Para desarrollar este paso, deberán seguirse las siguientes dos etapas.

\section{Etapa 2.1: Seleccionar expertos en los procesos y operaciones de la organización objeto de estudio}

Un experto es una persona que tiene un conocimiento especial respecto a un tema específico (Martino, 1993). El criterio clave para su selección es el nivel de conocimiento o grado de experticia en la temática objeto de investigación (Sourani \& Sohail, 2014), aunque según Martino (1993) otros criterios como la voluntad y la disponibilidad del candidato también revisten especial importancia. No se especifica en la literatura científica el número específico de expertos necesarios para la conducción de una técnica de expertos, sin embargo se reconoce que la cantidad mínima apropiada oscila entre siete u ocho (Sourani \& Sohail, 2014).

\section{Etapa 2.2: Desarrollar la matriz triangular de las relaciones cualitativas de adyacencia ideales (matriz $R$ )}

Con el objetivo de obtener las valoraciones de los expertos acerca de las relaciones cualitativas de adyacencia ideales entre cada par de centros de actividad, se realiza una reunión presencial. Tales valoraciones se registrarán mediante una escala ordinal donde E significa que la adyacencia es extremadamente importante, I importante, O ordinariamente importante, D indiferente y N indeseable. Se tratará de lograr un consenso respecto a los criterios que justifican dichas relaciones.

Una vez concluido el proceso, el moderador determinará el valor modal de las valoraciones para cada par bajo análisis y cuantificará la magnitud de las relaciones mediante un coeficiente $R_{i j}$ de la siguiente forma: $R_{i j}=10$ para relaciones de adyacencia extremadamente importantes; $R_{i j}=5$ para las importantes; $R_{i j}=2$ si tiene una importancia ordinaria; $R_{i j}=$ o si es indiferente, y por último, $R_{\mathrm{ij}}=-10$ si la adyacencia es indeseable. En caso de que la distribución de valoraciones sea multimodal, se escogerá la moda de menor coeficiente. Con esta información se desarrolla la matriz de relaciones cualitativas de adyacencia ideales, a la que alternativamente se le denominará matriz $R$.

$$
R=\left[\begin{array}{cccccc}
0 & r_{12} & r_{13} & r_{14} & \cdots & r_{1 n} \\
0 & 0 & r_{23} & r_{24} & \cdots & r_{2 n} \\
t_{31} & 0 & 0 & r_{34} & \cdots & r_{3 n} \\
0 & 0 & 0 & 0 & \cdots & r_{4 n} \\
\vdots & \vdots & \vdots & \vdots & \ddots & \vdots \\
0 & 0 & 0 & 0 & \cdots & 0
\end{array}\right]
$$

\section{Paso 3: Identificar las relaciones de adyacencia de la distribución espacial actual}

El coeficiente binario de adyacencia entre los centros de actividad i y j $\left(X_{i j}\right)$ tomará un valor de uno si los centros de actividad son 
adyacentes o cero si no lo son. Dos centros de actividad se consideran adyacentes cuando son contiguos o colindantes, o sea, cuanto tienen un límite fronterizo común, pudiendo ser este físico (un pasillo, una pared, etc.) o imaginario. Para cumplir con el principio de circulación y de la mínima distancia recorrida los departamentos con mayor intensidad de relaciones deben ser adyacentes. Como resultado de este paso del procedimiento se desarrolla la matriz $X$.

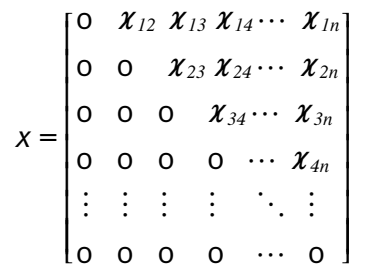

\section{Paso 4: Determinar el nivel de importancia relativa de los criterios cuantitativos frente a los criterios cualitativos}

Los criterios cuantitativos y los criterios cualitativos no necesariamente deben tener el mismo peso en la evaluación de un esquema de distribución espacial específico, por lo que requieren ser ponderados. En esta investigación, la ponderación o nivel de importancia relativa de los criterios cuantitativos respecto a los cualitativos es identificado con la letra griega alpha (a), y su valor depende de la naturaleza del sistema de producción objeto de estudio.

Al realizar un análisis de la distribución espacial en empresas de manufactura, se recomienda al analista emplear un valor de a superior a 0,5. De esta forma se estaría asignando un mayor peso o importancia relativa a las intensidades de transporte entre los centros de actividad respecto a los criterios de orden cualitativo. Esto se sustenta en la muy razonable idea de que al localizar los centros de actividad de mayor flujo de forma adyacente, se minimizan las distancias recorridas por el objeto de trabajo durante su proceso de transformación, y son precisamente estas actividades de manutención las que junto a las demoras no agregan valor al producto. De tal forma, mientras menor distancia recorra el objeto de trabajo, menor será su tiempo de procesamiento y mayores serán los índices de utilización de la maquinaria. En consecuencia, también la fuerza de trabajo mejoraría su aprovechamiento de la jornada laboral.
Todo lo anterior, impacta positivamente en el aumento de la productividad y en la reducción de los costos totales de producción. No obstante, puede ser que los analistas quieran considerar, dada su importancia, únicamente las intensidades de transporte como condicionante de las relaciones de adyacencia entre los centros de actividad, en cuyo caso a sería igual a la unidad.

En contraposición a los sistemas de manufactura, en los sistemas de servucción, generalmente, el flujo de materiales entre los distintos centros de actividad es irrelevante o inexistente. Así, los criterios cualitativos que justifican las relaciones entre los centros de actividad adquieren un rol preponderante en la decisión de organización espacial. Es muy probable que en este último caso, el analista le asigne a estos criterios una importancia relativa superior, por lo que a tomaría valores inferiores a 0,5, e incluso, en aquellos sistemas donde sólo existe flujo de información entre departamentos, esta ponderación puede estar muy cercana a cero.

\section{Paso 5: Calcular el Índice de Desempeño del Layout (IDL) e interpretar el resultado.}

El Índice de Desempeño del Layout (IDL) se calcula mediante la siguiente relación matemática:

$$
I D L=\frac{\alpha \cdot I f o+(1-\alpha) \cdot I f s}{100}
$$

Donde a representa el nivel de importancia relativa del flujo de trabajo (factor objetivo) respecto a los factores de cercanía subjetivos. Ifo es el Índice de flujo operativo entre los centros de actividad considerados en el estudio e Ifs representa el Índice de las relaciones subjetivas de adyacencia entre los centros de actividad.

El Ifo y el Ifs se formulan como sigue:

$$
\text { Ifo }=\frac{\sum_{i=1}^{n-1} \sum_{j=i+1}^{n}\left(\mathrm{~T}_{i j} \cdot \mathrm{x}_{i j}\right)}{\sum_{i=1}^{n-1} \sum_{j=i+1}^{n} \mathrm{~T}_{i j}} \cdot 100
$$

$$
I \mathrm{fS}=\frac{\sum_{i=1}^{n-1} \sum_{j=i+1}^{n}\left(R_{i j} \cdot x_{i j}\right)}{\sum_{i=1}^{n-1} \sum_{j=i+1}^{n} R_{i j}} \cdot 100
$$


$\mathrm{T}_{\mathrm{ij}}$ es la intensidad de transporte del objeto de trabajo entre el par de centros de actividad i y j. En el mismo caso, $X_{\mathrm{ij}}$ representa el coeficiente binario de adyacencia, y $R_{i j}$, por su parte, la valoración de los factores subjetivos de cercanía de acuerdo al juicio de expertos.

El Ifo representa el porcentaje del flujo total de materiales que está siendo transportado de forma óptima entre los centros de actividad, pues recorren la menor distancia posible dada su condición de adyacentes $\left(X_{i j}=1\right)$. Su complemento, (1- Ifo), permite cuantificar las reservas de mejora de la distribución espacial actual respecto a la solución óptima ideal, que supone adyacencia entre todos los pares de centros de actividad que tienen un flujo material distinto de cero entre sí (entiéndase óptima, pero no necesariamente factible desde el punto de vista geométrico).

El Ifs, por su parte, representa el nivel de bondad de la distribución actual de acuerdo con las relaciones de adyacencia ideales establecidas por el juicio de los expertos involucrados en el estudio. Este índice es particularmente importante cuando entre los centros de actividad, dada la naturaleza del sistema productivo en cuestión, no existe un flujo material tangible o este es irrelevante. De igual manera, su complemento (1- Ifs) reviste especial importancia, dado que constituye una medida de las reservas de mejora de la distribución espacial relacionada con el comportamiento actual de aquellos factores que no por ser subjetivos dejan de ser relevantes y que pueden impactar negativamente en el logro de la eficacia y la eficiencia de la organización.

El IDL, por su parte, permitirá identificar el posicionamiento de la distribución espacial bajo análisis, en un intervalo real que toma valores entre cero y uno. Un IDL cercano a cero indicará que el layout se acerca al anti-ideal o escenario más desfavorable, que es aquel donde los centros de actividad están dispuestos de forma caótica en el espacio fabril, sin ningún tipo de relación cualitativa y/o cuantitativa que justifique su adyacencia o contigüidad. Por su parte, un IDL cercano a la unidad, indicará que el layout se acerca a la mejor distribución espacial posible, es decir, a la óptima ideal, que supone adyacencia entre todos los centros de actividad que mantienen flujo de materiales entre sí, o tienen algún otro tipo de relación de naturaleza cualitativa.

Este posicionamiento entre el layout anti-ideal y el óptimo ideal, permite la identificación de oportunidades de mejora de la distribución en estudio y en consecuencia, reservas de mejora de la productividad del trabajo. Particularmente, si el IDL calculado fuese muy desfavorable (IDL<o.6), la gerencia podría iniciar estudios que permitan la generación de nuevas alternativas de layout, e implementar aquella variante que cumpla con las restricciones de espacio y de capital, a la vez que mejore el Índice de Desempeño del Layout.

\section{ESTUDIO DE CASO}

En este apartado se muestra la aplicación del Índice de Desempeño del Layout propuesto, en un caso real. La empresa pertenece al sector metalmecánico de la ciudad de Guayaquil y se dedica a la construcción de bombas de flujo axial, turbinas, compresores, transportadores de banda y matricería para las empresas procesadoras y envasadoras de camarón de la región costeña del Ecuador.

Mediante entrevistas y observación directa se detectaron los siguientes centros de actividad, los cuales han sido enumerados tal como se presenta a continuación:

1. Taller de prensas

2. Área de pulido

3. Taller de roscado

4. Zona de arenado

5. Taller de fresado

6. Módulo de control numérico computarizado

7. Taller de tornería

8. Área de montaje

9. Área de inspección y embalaje

10. Área de recepción y despacho

11. Oficinas

12. Almacén

Mediante los reportes de operaciones que maneja la organización, se determinaron las intensidades de transporte que se presentaron durante el año 2014 expresadas en toneladas anuales ( $\mathrm{t} / \mathrm{año}$ ). Con esta información se desarrolló la matriz $T$ que se presenta a continuación. 


$$
t=\left[\begin{array}{cccccccccccc}
0 & 0 & 10,2 & 0 & 0 & 0 & 15,7 & 0 & 0 & 0 & 0 & 0 \\
0 & 0 & 0 & 0 & 0 & 0 & 0 & 31,6 & 12,8 & 0 & 0 & 0 \\
0 & 0 & 0 & 0 & 0 & 13,5 & 0 & 5,9 & 2,3 & 0 & 0 & 0 \\
0 & 0 & 0 & 0 & 0 & 23,1 & 26,5 & 0 & 0 & 0 & 0 & 0 \\
0 & 2,5 & 1,6 & 0 & 0 & 0 & 0 & 4,1 & 0 & 0 & 0 & 0 \\
0 & 18,2 & 0 & 0 & 0 & 0 & 3,4 & 12,2 & 0 & 0 & 0 & 0 \\
0 & 19,8 & 8,9 & 0 & 6,4 & 0 & 0 & 0 & 0 & 0 & 0 & 0 \\
0 & 0 & 0 & 0 & 0 & 0 & 0 & 0 & 30,2 & 0 & 0 & 0 \\
0 & 1,2 & 0 & 0 & 0 & 0 & 0 & 0 & 0 & 0 & 0 & 39,3 \\
0 & 0 & 0 & 0 & 0 & 0 & 0 & 0 & 0 & 0 & 0 & 43,4 \\
0 & 0 & 0 & 0 & 0 & 0 & 0 & 0 & 0 & 0 & 0 & 0 \\
12,3 & 0 & 0 & 10,8 & 22,1 & 33,4 & 38,7 & 0 & 0 & 0 & 0 & 0
\end{array}\right]
$$

Con la matriz $\mathrm{T}$ como insumo, aplicando la relación $\mathrm{T}_{\mathrm{ji}}=\mathrm{t}_{\mathrm{ij}}+\mathrm{t}_{\mathrm{ji}}$, pudo obtenerse la matriz $\mathrm{T}$, que es la matriz triangular no orientada de las intensidades de transporte totales. La misma que considera el flujo de ida y vuelta, en caso de existir, entre cada par de centros de actividad.

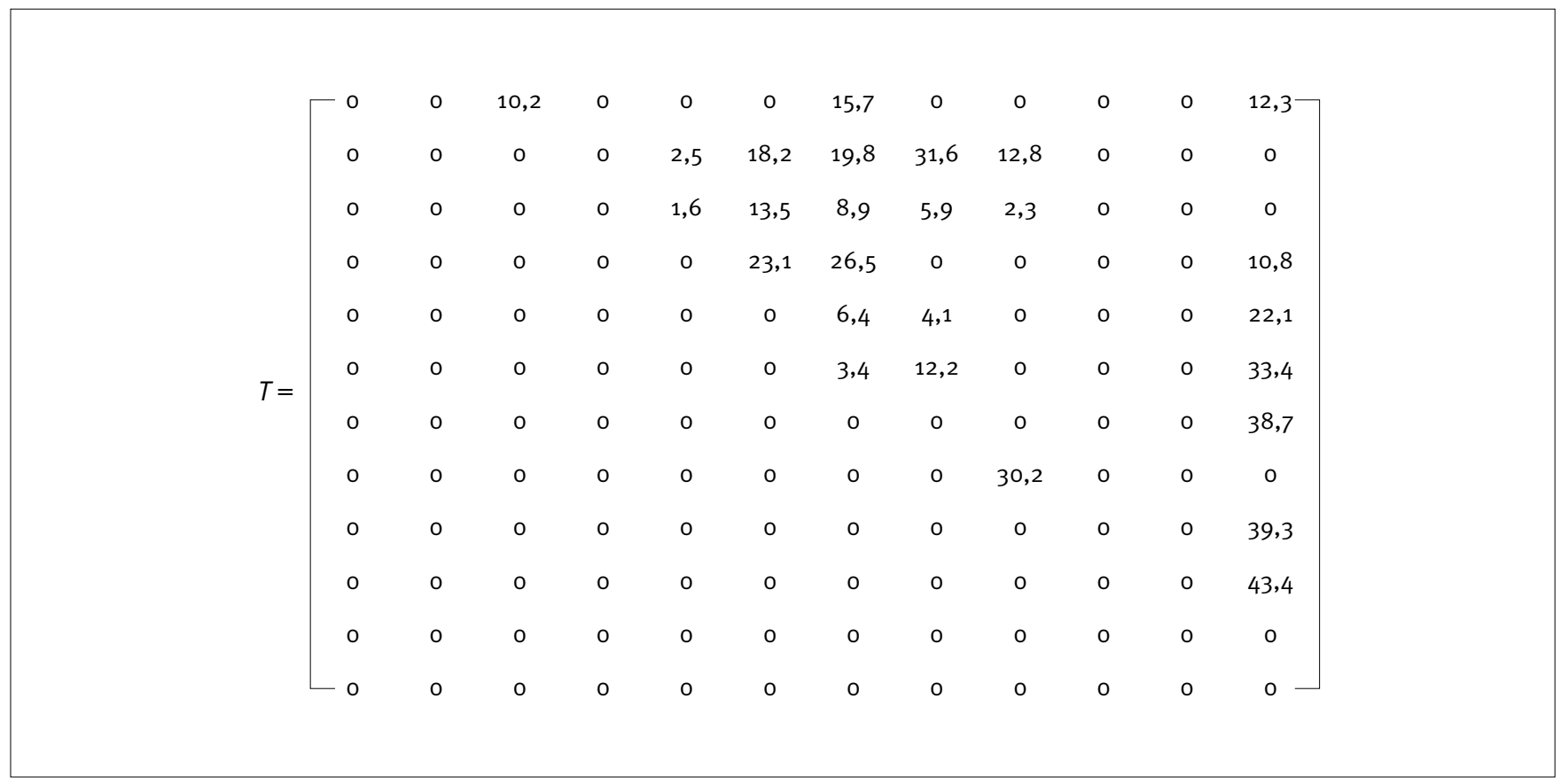

A continuación se hizo una selección de ocho expertos, todos trabajadores de la organización y con más de cinco años de experiencia. Esto permitió asegurar que la totalidad de candidatos conocieran a fondo los procesos que tienen lugar en la empresa. A partir de sus valoraciones acerca de las relaciones cualitativas de adyacencia ideales entre cada par de centros de actividad se obtuvo la matriz R. 


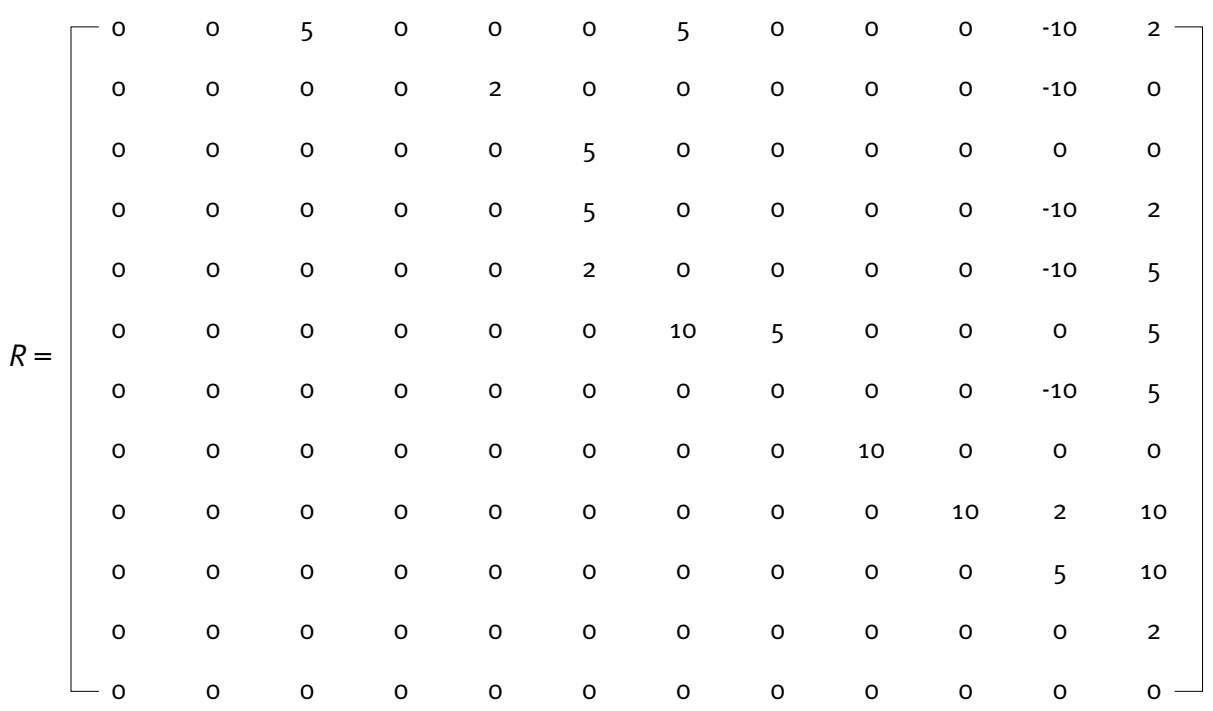

Un resumen del proceso de obtención de las relaciones cualitativas de adyacencia ideales mediante el juicio de los expertos puede observarse en la Tabla 1. En este caso, para facilitar la representación, se excluyen las relaciones de importancia tipo D (indiferente) que determinan coeficientes $R_{i j}=0$.

Tabla 1. Resumen del proceso de obtención de las relaciones cualitativas de adyacencia mediante el juicio de expertos

\begin{tabular}{|c|c|c|c|c|c|c|c|c|c|c|c|c|}
\hline \multicolumn{2}{|c|}{$\begin{array}{l}\text { Centros de } \\
\text { actividad }\end{array}$} & \multicolumn{8}{|c|}{ Relaciones ideales de adyacencia } & \multirow{2}{*}{ Moda } & \multirow{2}{*}{$R_{i j}$} & \multirow{2}{*}{ Justificación } \\
\hline $\mathbf{i}$ & j & $E_{1}$ & $\mathrm{E}_{2}$ & $\mathrm{E}_{3}$ & $\mathrm{E}_{4}$ & $E_{5}$ & $E_{6}$ & $E_{7}$ & $E_{8}$ & & & \\
\hline 1 & 3 & I & I & $\mathrm{E}$ & 0 & $\mathrm{E}$ & I & I & 1 & I & 5 & Facilitar la supervisión \\
\hline 1 & 7 & 0 & I & 1 & I & 0 & I & I & I & I & 5 & Comparten personal común \\
\hline 1 & 12 & I & 0 & 0 & I & 0 & 0 & 0 & I & 0 & 2 & Reducir traslado de materia prima pesada \\
\hline 2 & 5 & 0 & 0 & 0 & 0 & I & I & I & 0 & 0 & 2 & Facilitar la supervisión \\
\hline 2 & 11 & $\mathrm{~N}$ & $\mathrm{~N}$ & $\mathrm{~N}$ & D & $\mathrm{N}$ & D & $\mathrm{N}$ & $\mathrm{N}$ & $\mathrm{N}$ & -10 & Alto nivel de ruido \\
\hline 3 & 6 & 0 & I & I & I & 0 & 0 & I & D & I & 5 & Facilitar la supervisión \\
\hline 4 & 7 & I & 0 & I & I & I & 0 & 1 & I & I & 5 & Comparten personal común \\
\hline 4 & 11 & $\mathrm{~N}$ & $\mathrm{~N}$ & $\mathrm{~N}$ & $\mathrm{~N}$ & D & $\mathrm{N}$ & $\mathrm{N}$ & D & $\mathrm{N}$ & -10 & Aire enrarecido (polvos) \\
\hline 4 & 12 & D & 0 & I & 0 & 0 & 0 & D & 0 & 0 & 2 & Aire enrarecido (polvos) \\
\hline 5 & 6 & 0 & 0 & 0 & D & D & 0 & D & 0 & 0 & 2 & Facilitar la supervisión \\
\hline 5 & 11 & D & $\mathrm{N}$ & $\mathrm{N}$ & $\mathrm{N}$ & $\mathrm{N}$ & $\mathrm{N}$ & $\mathrm{N}$ & $\mathrm{N}$ & $\mathrm{N}$ & -10 & $\begin{array}{l}\text { Ruido y posible proyección de virutas o } \\
\text { limallas }\end{array}$ \\
\hline 5 & 12 & 0 & 1 & I & I & 0 & I & I & I & 1 & 5 & Facilitar control de trabajo en proceso \\
\hline
\end{tabular}


(continuación) Tabla 1. Resumen del proceso de obtención de las relaciones cualitativas de adyacencia mediante el juicio de expertos

\begin{tabular}{|c|c|c|c|c|c|c|c|c|c|c|c|c|}
\hline \multicolumn{2}{|c|}{$\begin{array}{l}\text { Centros de } \\
\text { actividad }\end{array}$} & \multicolumn{8}{|c|}{ Relaciones ideales de adyacencia } & \multirow{2}{*}{ Moda } & \multirow{2}{*}{$R_{i j}$} & \multirow{2}{*}{ Justificación } \\
\hline i & j & $E_{1}$ & $\mathrm{E}_{2}$ & $E_{3}$ & $\mathrm{E}_{4}$ & $E_{5}$ & $E_{6}$ & $\mathrm{E}_{7}$ & $\mathrm{E}_{8}$ & & & \\
\hline 6 & 7 & $\mathrm{E}$ & I & $\mathrm{E}$ & $\mathrm{E}$ & $\mathrm{E}$ & I & $E$ & $E$ & E & 10 & Comparten personal común \\
\hline 6 & 8 & I & E & I & I & I & I & I & 0 & I & 5 & Actividades con flujo directo \\
\hline 7 & 11 & $\mathrm{~N}$ & D & $\mathrm{N}$ & $\mathrm{N}$ & $\mathrm{N}$ & D & $\mathrm{N}$ & $\mathrm{N}$ & $\mathrm{N}$ & -10 & $\begin{array}{l}\text { Ruido y posible proyección de virutas o } \\
\text { limallas }\end{array}$ \\
\hline 7 & 12 & 0 & 0 & I & 0 & 0 & I & 0 & I & I & 5 & Facilitar control de trabajo en proceso \\
\hline 8 & 9 & $\mathrm{E}$ & $\mathrm{E}$ & $\mathrm{E}$ & I & I & $\mathrm{E}$ & $\mathrm{E}$ & $\mathrm{E}$ & $\mathrm{E}$ & 10 & Actividades con flujo directo \\
\hline 9 & 12 & $\mathrm{E}$ & $\mathrm{E}$ & $\mathrm{E}$ & I & $\mathrm{E}$ & $\mathrm{E}$ & 1 & $\mathrm{E}$ & $\mathrm{E}$ & 10 & Agilizar estiba a bodega y facilitar el control \\
\hline 10 & 11 & 0 & I & I & 0 & I & 0 & I & I & I & 5 & Comparten personal común \\
\hline 10 & 12 & I & $\mathrm{E}$ & $\mathrm{E}$ & $\mathrm{E}$ & $\mathrm{E}$ & $\mathrm{E}$ & I & $\mathrm{E}$ & $E$ & 10 & $\begin{array}{l}\text { Facilitar el control de la recepción y el } \\
\text { despacho }\end{array}$ \\
\hline 11 & 12 & 0 & 0 & D & 0 & 0 & D & D & 0 & 0 & 2 & $\begin{array}{l}\text { Facilitar la recepción de materiales y su } \\
\text { control }\end{array}$ \\
\hline
\end{tabular}

La matriz X se obtuvo mediante observación directa, y representa las relaciones de adyacencia entre los centros de actividad de acuerdo a la distribución espacial actual.

$X=\left[\begin{array}{llllllllllll}0 & 0 & 1 & 1 & 0 & 0 & 0 & 1 & 0 & 0 & 1 & 0 \\ 0 & 0 & 1 & 0 & 0 & 0 & 0 & 0 & 1 & 1 & 0 & 0 \\ 0 & 0 & 0 & 1 & 0 & 0 & 0 & 1 & 1 & 0 & 0 & 0 \\ 0 & 0 & 0 & 0 & 0 & 0 & 0 & 0 & 0 & 0 & 0 & 0 \\ 0 & 0 & 0 & 0 & 0 & 1 & 1 & 0 & 1 & 0 & 0 & 1 \\ 0 & 0 & 0 & 0 & 0 & 0 & 1 & 0 & 0 & 0 & 1 & 0 \\ 0 & 0 & 0 & 0 & 0 & 0 & 0 & 1 & 1 & 0 & 1 & 0 \\ 0 & 0 & 0 & 0 & 0 & 0 & 0 & 0 & 1 & 0 & 0 & 0 \\ 0 & 0 & 0 & 0 & 0 & 0 & 0 & 0 & 0 & 1 & 0 & 1 \\ 0 & 0 & 0 & 0 & 0 & 0 & 0 & 0 & 0 & 0 & 0 & 1 \\ 0 & 0 & 0 & 0 & 0 & 0 & 0 & 0 & 0 & 0 & 0 & 1 \\ 0 & 0 & 0 & 0 & 0 & 0 & 0 & 0 & 0 & 0 & 0 & 0\end{array}\right]$

Una vez realizados los cálculos, considerando una importancia relativa del flujo de trabajo sobre los factores cualitativos del $75 \%(a=0,75)$, el índice de flujo operativo obtuvo un valor de $39,38 \%$. Esto implica que el flujo de trabajo en la distribución espacial actual está muy alejado del escenario óptimo, específicamente en un 60,62\%. El layout actual no cumple con el principio de circulación ni el de la mínima distancia recorrida posible, por lo que admite mejoras.

El Ifs obtuvo un valor de $68,75 \%$. De ahí se interpreta que el layout actual también está alejado de la variante de ordenamiento espacial ideal establecida por el juicio de los expertos. Al comparar este valor con el del Ifo, puede deducirse que la distribución espacial actual está sobreestimando el comportamiento de los factores cualitativos respecto al flujo del objeto de trabajo, aun cuando el nivel de importancia relativa de este último es tres veces mayor $(a=0,75)$, por tener una mayor contribución a la reducción de costos e impactar en mayor medida sobre el mejoramiento de la productividad del trabajo.

Los valores obtenidos por el Ifo y el Ifs hacen que el IDL esté muy bajo (IDL = 46,72\%), lo cual significa que el sistema productivo objeto de estudio tiene unas reservas de mejora de su layout del 53,28\% (1-IDL×100 =53,28\%). Ante esta situación, una redistribución espacial es inminente para optimizar el tiempo de fabricación y los costos, aumentar la productividad y contribuir a la competitividad de la organización. Es importante que en la redistribución a realizar se prioricen aquellos cambios que impacten positivamente en el índice de desempeño, siempre que lo permitan las restricciones de espacio y de capital. 


\section{DISCUSIÓN}

En la generación y evaluación de alternativas de layout mediante el SLP (Muther, 1961), criterios cuantitativos como el flujo de materiales tienen el mismo tratamiento que los criterios de naturaleza cualitativa. Ambos tipos de factores, necesarios al intentar justificar el nivel de cercanía entre centros de actividad, son medidos con la misma escala ordinal, donde $A=$ Absolutamente necesaria, $\mathrm{E}=$ Especialmente importante, $\mathrm{I}=$ Importante, $\mathrm{O}=$ Ordinariamente importante, $\mathrm{U}=$ No importante $\mathrm{y} X=$ Indeseable.

Por otra parte, AHP, que es una de las herramientas de decisión multicriterio más utilizadas en la evaluación de alternativas de layout, utiliza la misma escala de nueve puntos para las valoraciones de importancia de ambos tipos de criterios, lo cual hace difícil clasificar particularmente a los criterios de tipo cuantitativo. Por su parte, el indicador propuesto en este estudio (IDL) discrimina ambos tipos de criterios y permite al proyectista decidir cuál de ellos debe tener una mayor repercusión en la decisión final mediante el coeficiente a, que representa el nivel de importancia relativa de los criterios objetivos respecto a los subjetivos.

En la literatura, la evaluación de layouts ha sido abordada de forma frecuente mediante métodos de decisión multicriterio (Arunkumar, Barnabas, Kumar, \& Kamatchi, 2012; Emami \& Nookabadi, 2013; Gao et al., 2010; Nagapak \& Phruksaphanrat, 2011; Önüt et al., 2008; Shahin \& Poormostafa, 2011; Tortorella \& Fogliatto, 2008; Yang \& Deuse, 2012; Yang, Deuse, \& Jiang, 2013; Yang \& Kuo, 2003; Yang et al., 2000). Sin embargo, tales métodos ofrecen únicamente medidas relativas que solo tienen sentido si se comparan varias alternativas, por tanto, no son de utilidad para evaluar el desempeño del layout actual de una planta industrial, o lo que es lo mismo, no permiten diagnosticar la necesidad de una redistribución.

Solo Neumann y Fogliatto (2013) propusieron un índice para la evaluación del layout existente, al que denominaron IGFL. Esta herramienta se enfoca en la medición de la flexibilidad del layout y permite identificar sus oportunidades de mejora. Sin embargo, emplea un conjunto excesivo de factores de evaluación (tres factores primarios, 12 secundarios, 33 terciarios y 50 indicadores) que al decir de sus propios autores no pueden ser cambiados por el analista. Tampoco es aconsejado por estos autores el cambio de las intensidades de relacionamiento entre los factores terciarios y los indicadores. Además, la totalidad de factores bajo análisis no necesariamente son relevantes o aplicables a cualquier sistema de producción fabril. Incluso aquellos factores terciarios relacionados con el flujo de materiales son tratados cualitativamente, desaprovechando la magnitud del flujo de producción que transita a lo largo de todo el sistema de producción.

El IDL, por su parte, permite evaluar el desempeño de la distribución espacial actual de una planta existente, con una distribución espacial óptima ideal (no necesariamente factible desde el punto de vista geométrico), que considera adyacencia entre todos aquellos centros de actividad que tienen flujo material entre sí (medido cuantitativamente) y que, a su vez, satisfacen criterios cualitativos relevantes establecidos mediante el juicio de expertos. Tales criterios cualitativos varían de acuerdo al sistema de producción considerado y al nivel de conocimiento de los expertos acerca de los procesos y las operaciones que se desarrollan en la organización. Como resultado, el IDL permite conocer el posicionamiento del layout bajo análisis, en un intervalo real que parte de la situación más desfavorable (layout anti-ideal, donde IDL $=0$ ) hasta la mejor organización espacial posible (layout óptimo ideal, donde IDL =1), permitiendo la identificación de oportunidades de mejora. Incluso el IDL podría utilizarse también como un método de evaluación alternativo a los referenciados en la literatura, para la evaluación y selección de la mejor distribución espacial entre un grupo de alternativas obtenidas mediante cualquier método de generación de layouts.

Un valor de IDL inferior a o,6 es un indicativo de la necesidad de una redistribución espacial de la planta objeto de estudio, pues evidentemente las actividades con mayor flujo de materiales no están lo más próximas posible (incumpliendo el principio de la mínima distancia recorrida), ni alineadas de acuerdo a la secuencia con la que se tratan, elaboran o montan los materiales (principio de la circulación). De tal manera, puede afirmarse que el Índice de Desempeño del Layout aquí desarrollado puede sustentar la toma de decisiones relativas al reordenamiento o redistribución espacial de los centros de actividad de una organización, permitiendo la priorización de aquellos ajustes que involucren menores desembolsos de dinero y que tengan un mayor impacto positivo en la productividad del sistema.

Es importante destacar que esta investigación no cubre la determinación de las oportunidades de mejora de la organización espacial de los factores productivos a nivel de detalle dentro de cada centro de actividad, quedando pendiente dicho análisis para futuras investigaciones.

\section{CONCLUSIONES}

Esta investigación presentó el procedimiento para el cálculo de un Índice de Desempeño del Layout (IDL) que permite evaluar el desempeño de la distribución espacial de sistemas de producción e identificar sus reservas de mejora. Dicho indicador, en esencia, permite determinar el posicionamiento del layout bajo análisis entre el escenario ideal y anti-ideal, basado en el comportamiento simultáneo de factores cuantitativos y cualitativos de acuerdo al contexto práctico específico del sistema productivo considerado. 
Su aplicación como caso de estudio en una empresa del sector metalmecánico permitió identificar reservas de mejora de la distribución espacial del 53,28\%. Este resultado sustenta la necesidad inminente de una redistribución espacial de la planta objeto de estudio, pues el layout actual está más cercano al escenario anti-ideal que al óptimo ideal.

\section{REFERENCIAS}

Apple, J. M. (1977). Plant layout and material handling. New York, EEUU: John Wiley \& Sons.

Ariafar, S., Ismail, N., Tang, S. H., Ariffin, M. K. A. M., \& Firoozi, Z. (2011). Facility layout design for hybrid cellular manufacturing systems. International Journal of Physical Sciences, 6(14), 3551-3556. doi:10.5897/ijps11.1012

Armour, G. C., \& Buffa, E. S. (1963). A heuristic algorithm and simulation approach to relative location of facilities. Management Science, 9(2), 294-309.

Arunkumar, N., Barnabas, S. G., Kumar, N. D., \& Kamatchi, T. (2012). Facility layout selection for the blood inventory using PROMETHEE II method. International Journal of Engineering Research and Applications, 2(4), 1161-1166.

Askin, R. G., \& Standridge, C. R. (1993). Modeling and analysis of manufacturing systems. New York, EEUU: Wiley.

Bazaraa, M. S. (1975). Computerized layout design: A branch and bound approach. AlIE Transactions, 7(4), 432-438. doi:10.1080/05695557508975028

Bendaña, R., Caño, A. Del, \& Cruz, M. P. De La. (2008). Contractor selection: Fuzzy control approach. Canadian Journal of Civil Engineering, 35(5), 473-486. foi:10.1139/Lo7-127

Bozer, Y. A., Meller, R. D., \& Erlebacher, S. J. (1994). An improvement type layout algorithm for single and multiple-floor facilities. Management Science, 40(7), 918-932.

Bradley, L., \& Stewart, K. (2002). A Delphi study of the drivers and inhibitors of internet banking. International Journal of Bank Marketing, 20(6), 250-260. doi:10.1108/02652320210446715

Buffa E.S. (1955). Sequence analysis for functional layouts. Journal of Industrial Engineering, 6, 12-25.

Chase, R. B., \& Jacobs, F. R. (2014). Administración de operaciones, producción y cadena de suministros (13 ${ }^{\mathrm{a}}$. ed.). Ciudad de México, México: McGraw Hill.

Diego-Mas, J. A. (2006). Optimización de la distribución en planta de instalaciones industriales mediante algoritmos genéticos: Aportación al control de la geometría de las actividades (Tesis de Doctorado en Ingeniería Industrial, Universidad Politécnica de Valencia, Valencia, España).

Drira, A., Pierreval, H., \& Hajri-Gabouj, S. (2007). Facility layout problems: A survey. Annual Reviews in Control, 31(2), 255-267. doi:10.1016/j. arcontrol.2007.04.001

Edis, R. S., Kahraman, B., Araz, O. U., \& Özfirat, M. K. (2011). A facility layout problem in a marble factory via simulation. Mathematical and Computational Applications, 16(1), 97-104.
Emami, S., \& Nookabadi, A. S. (2013). Managing a new multi-objective model for the dynamic facility layout problem. The International Journal of Advanced Manufacturing Technology, 68(9), 2215-2228. doi:10.1007/s00170-013-4820-5

Foulds, L. R., \& Robinson, D. F. (1978). Graph theoretic heuristics for the plant layout problem. The International Journal of Production Research, 16(1), 27-37. doi:10.1080/00207547808929997

Francis, R. L., McGinnis, L. F., \& White, J. A. (1992). Facility layout and location: An analytical approach. New Jersey. EEUU: Prentice Hall.

Fu, M. C., \& Kaku, B. K. (1997). Minimizing work-in-process and material handling in the facilities layout problem. IIE Transactions, 29(1), 29-36. doi:10.1080/07408179708966309

Gao, Z., Yoshimoto, K., \& Ohmori, S. (2010). Application of AHP/DEA to facility layout selection. Proceedings of the III International Joint Conference on Computational Science and Optimization, IEEE Computer Society, Huangshan, EEUU.

García-Hernandez, L., Arauzo-Azofra, A., Salas-Morera, L., Pierreval, H., \& Corchado, E. (2013). Recycling plants layout design by means of an interactive genetic algorithm. Intelligent Automation and Soft Computing, 19(3), 457-468. doi:10.1080/10798587.2013.803679

Garey, M. R., \& Johnson, D. S. (1979). Computers and intractability: A guide to the theory of NP-completeness. San Francisco, EEUU: Freeman.

Han, K. H., Bae, S. M., \& Jeong, D. M. (2012). A decision support system for facility layout changes. Proceedings of the VII WSEAS International Conference, Recent Advances In Computer Engineering Series, Vienna, Austria. Retrieved from http://www.wseas.us/e-library/ conferences/2012/Vienna/COMPUTERS/COMPUTERS-11.pdf

Hillier, F. S., \& Connors, M. M. (1966). Quadratic assignment problem algorithms and the location of indivisible facilities. Management Science, 13(1), 42-57. doi:10.1287/mnsc.13.1.42

Immer, R. J. (1953). Materials Handling. New York, EEUU: McGraw-Hill.

Kanaganayagam, K., Muthuswamy, S., \& Damodaran, P. (2015). Lean methodologies to improve assembly line efficiency: An industrial application. International Journal of Industrial and Systems Engineering, 20(1), 104-116. doi:10.1504/IJISE.2015.069000

Kaufman, L., \& Broeckx, F. (1978). An algorithm for the quadratic assignment problem using Bender's decomposition. European Journal of Operational Research, 2(3), 207-211. doi:10.1016/0377$2217(78) 90095-4$

Koopmans, T. C., \& Beckman, M. (1957). Assignment problems and the location of economic activities. Econometrica, 25(1), 53-76. doi:10.2307/1907742

Kotler, P., \& Keller, K. L. (2012). Dirección de marketing (14ª ed.). Madrid, España: Addison-Wesley.

Krajewski, L. J., Malhotra, M. K., \& Ritzman, L. P. (2015). Operations management: Processes and supply chains. New York, EEUU: Prentice Hall.

Kulturel-Konak, S. (2007). Approaches to uncertainties in facility layout problems: Perspectives at the beginning of 21st century. Journal of Intelligent Manufacturing, 18(2), 273-284. doi:10.1007/s10845-0070020-1

Kusiak, A., \& Heragu, S. S. (1987). The facility layout problem. European Journal of Operational Research, 29(3), 229-251. doi:10.1016/0377$2217(87) 90238-4$

Lawler, E. L. (1963). The quadratic assignment problem. Management Science, 9(4), 586-599. doi:10.1287/mnsc.9.4.586 
Lee, R., \& Moore, J. M. (1967). Corelap-computerized relationship layout planning. Journal of Industrial Engineering, 18, 195-200.

Lucko, G., \& Rojas, E. M. (2010). Research validation: Challenges and opportunities in the construction domain. Journal of Construction Engineering and Management, 136(1), 127-135. doi:10.1061/(asce) c0.1943-7862.0000025

Mallikarjuna, K., Veeranna, V., \& Reddy, K. H. C. (2011). FMS layout design by genetic algorithm and tabu search. International Journal of Applied Engineering Research, 6(14), 1767-1776.

Martino, J. P. (1993). Technological forecasting for decision making (3rd. ed.). New York, EEUU: McGraw Hill.

Matai, R., Singh, S. P., \& Mittal, M. L. (2010). Facility layout problem: A state-of-the-art review. Vilakshan: The XIMB Journal of Management, 7(2), 81-106.

McKendall, J. A. R., Shang, J., \& Kuppussamy, S. Y. (2006). Simulated annealing heuristics for the dynamic facility layout problem. Computers \& Operational Research, 33(8), 2431-2444. doi:10.1016/j. cor.2005.02.021

Mecklenburgh, J. C. (1985). Process plant layout. New York, EEUU: Longman.

Meller, R. D., \& Bozer, Y. A. (1996). A new simulated annealing algorithm for the facility layout problem. International Journal of Production Research, 34(6), 1675-1692. doi:10.1080/00207549608904990

Meller, R. D., \& Gau, K. Y. (1996). The facility layout problem: Recent and emerging trends and perspectives. Journal of Manufacturing Systems, 15(5), 351-366. doi:10.1016/0278-6125(96)84198-7

Moslemipour, G., Lee, T. S., \& Rilling, D. (2012). A review of intelligent approaches for designing dynamic and robust layouts in flexible manufacturing systems. The International Journal of Advanced Manufacturing Technology, 6o(1), 11-27. doi:10.1007/s00170-011-3614-x

Muther, R. (1961). Systematic layout planning. Boston, EEUU: Industrial Education Institute.

Nadler, G. (1967). Work systems design: The IDEALS concept. Homewood, EEUU: Richard D. Irwin.

Nagapak, N., \& Phruksaphanrat, B. (2011). Cellular manufacturing layout design and selection: A case study of electronic manufacturing service plant. Proceedings of the International Multi Conference of Engineers and Computer Scientists, IMECS, Hong Kong, China. Recuperado de http://iaeng.org/publication/IMECS2011/IMECS2011_pp1182-1187.pdf

Neumann, C. S. R., \& Fogliatto, F. S. (2013). Sistemática para avaliação e melhoria da flexibilidade de layout em ambientes dinâmicos. Gestão \& Produção, 20(2), 235-254. doi:10.1590/S0104$530 X 2013000200001$

Önüt, S., Kara, S. S., \& Efendigil, T. (2008). A hybrid fuzzy MCDM approach to machine tool selection. Journal of Intelligent Manufacturing, 19(4), 443-453. doi:10.1007/s10845-008-0095-3

Orndoff, C. J. (2005). Promising new tool for stakeholder interaction. Journal of Architectural Engineering, 11(4), 139-146. doi:10.1061/ (asce)1076-0431(2005)11:4(139)

Padel, S., \& Midmore, P. (2005). The development of the European market for organic products: Insights from a Delphi study. British Food Journal, 107(8), 626-646. doi:10.1108/00070700510611011

Río-Cidoncha, G. Del, Martínez-Palacios, J., \& Iglesias, J. E. (2007). A multidisciplinary model for floorplan design. International Journal of Production Research, 45(15), 3457-3476.
Reed, R. (1961). Plant layout: Factors, principles and techniques. Homewood, EEUU: Richard D. Irwin.

Ripon, K. S. N., \& Torresen, J. (2014). Integrated job shop scheduling and layout planning: A hybrid evolutionary method for optimizing multiple objectives. Evolving Systems, 5(2), 121-132. doi:10.1007/ S12530-013-9092-7

Sadeghpour, F., \& Andayesh, M. (2015). The constructs of site layout modeling: An overview. Canadian Journal of Civil Engineering, 42(3), 199-212. doi:10.1139/cjce-2014-0303

Seehof, J. M., \& Evans, W. O. (1967). Automated layout design program. Journal of Industrial Engineering, 18(2), 690-695.

Shahin, A., \& Poormostafa, M. (2011). Facility layout simulation and optimization: An integration of advanced quality and decision making tools and techniques. Modern Applied Science, 5(4), 95-111. doi:10.5539/mas.v5n4p95

Sharma, P., Singh, R. P., \& Singhal, S. (2013). A review of meta-heuristic approaches to solve facility layout problem. International Journal of Emerging Research in Management \& Technology, 2(10), 29-33.

Singh, S. P., \& Sharma, R. R. (2006). A review of different approaches to the facility layout problems. The International Journal of Advanced Manufacturing Technology, 30(5), 425-433. doi:10.1007/s00170005-0087-9

Sourani, A., \& Sohail, M. (2014). The Delphi method: Review and use in construction management research. International Journal of Construction Education and Research, 11(1), 54-76. doi:10.1080/155 78771.2014.917132

Suo, X. (2012). Facility layout. In F. A. Aziz (Ed.), Manufacturing system (In Tech, pp. 405-432). Recuperado de http://www.intechopen.com/ books/manufacturing-system/facility-layout

Tortorella, G. L., \& Fogliatto, F. S. (2008). Planejamento sistemático de layout com apoio de análise de decisão multicritério. Revista Produção, 18(3), 609-624. doi:10.1590/S0103-65132008000300015

Vasudevan, K., \& Son, Y.J. (2011). Concurrent consideration of evacuation safety and productivity in manufacturing facility planning using multi-paradigm simulations. Computers and Industrial Engineering, 61(4), 1135-1148. doi:10.1016/j.cie.2011.07.003

Yang, L., \& Deuse, J. (2012). Multiple-attribute decision making for an energy efficient facility layout design. Proceedings.of the XLV CIRP Conference on Manufacturing Systems, Athens, Greece.

Yang, L., Deuse, J., \& Jiang, P. (2013). Multiple-attribute decisionmaking approach for an energy-efficient facility layout design. The International Journal of Advanced Manufacturing Technology, 66(5), 795-807. doi:10.1007/s00170-012-4367-x

Yang, T., \& Kuo, C. (2003). A hierarchical AHP/DEA methodology for the facilities layout design problem. European Journal of Operational Research, 147(1), 128-136. doi:10.1016/S0377-2217(02)00251-5

Yang, T., Su, C. T., \& Hsu, Y. R. (2000). Systematic layout planning: A study on semiconductor wafer fabrication facilities. International Journal of Operations \& Production Management, 20(11), 1359-1371. doi:10.1108/01443570010348299

Yeung, J. F., Chan, A. P., \& Chan, D. W. (2009). Developing a performance index for relationship-based construction projects in Australia: Delphi study. Journal of Management in Engineering, 25(2), 59-68. doi:10.1061/(asce)0742-597x(2009)25:2(59) 